\title{
Derandomizing HSSW Algorithm for 3-SAT
}

\author{
Kazuhisa Makino ${ }^{1}$, Suguru Tamaki ${ }^{2}$, and Masaki Yamamoto ${ }^{3}$ \\ 1 Graduate School of Information Science and Technology, University of Tokyo \\ makino@mist.i.u-tokyo.ac.jp \\ 2 Graduate School of Informatics, Kyoto University \\ tamak@kuis.kyoto-u.ac.jp \\ 3 Dept. of Informatics, Kwansei-Gakuin University \\ masaki.yamamoto@kwansei.ac.jp
}

\begin{abstract}
We present a (full) derandomization of HSSW algorithm for 3-SAT, proposed by Hofmeister, Schöning, Schuler, and Watanabe in [STACS'02]. Thereby, we obtain an $\widetilde{O}\left(1.3303^{n}\right)$-time deterministic algorithm for 3-SAT, which is currently fastest.
\end{abstract}

\section{Introduction}

The satisfiability problem (SAT) is one of the most fundamental NP-hard problems. Questing for faster (exponential-time) exact algorithms is one of the main research directions on SAT. Initiated by Monien and Speckenmeyer [12], a number of algorithms for exactly solving SAT have been proposed, and many important techniques to analyze those algorithms have been developed [6]. See also $[5,13,15,16,20]$, for example. The most well-studied restriction of the satisfiability problem is 3-SAT $[2,3,7-10,18,19,21]$, i.e., the CNF satisfiability problem with clauses of length at most three. The currently best known time complexities for 3-SAT are $\widetilde{O}\left(1.3211^{n}\right)$ achieved by randomized algorithms [7] and $\widetilde{O}\left(1.3334^{n}\right)$ derived by deterministic algorithms [13], where $n$ denotes the number of Boolean variables in the formula.

As we can see, there is a noticeable gap between the current randomized and deterministic time bounds for 3-SAT. This raises a natural question: Can we close the gap completely? One promising way to attack the above question is derandomization. Roughly speaking, the task of derandomization is to construct an algorithm which deterministically and efficiently simulates the original randomized algorithm. There are a lot of strong derandomization results, e.g. $[1,4,14,17]$ to name a few, and one excellent example in the area of satisfiability is the derandomization of Schöning's algorithm for $k$-SAT.

In [20], Schöning proposed a simple randomized local search algorithm for $k$-SAT, and showed that it runs in expected time $\widetilde{O}\left((2-2 / k)^{n}\right)$, which is $\widetilde{O}\left(1.3334^{n}\right)$ when $k=3$. Later it was derandomized by Dantsin et al. [5]. They proposed a $k$-SAT algorithm that deterministically simulates Schöning's algorithm in time $\widetilde{O}\left((2-2 /(k+1))^{n}\right)$, which is $\widetilde{O}\left(1.5^{n}\right)$ when $k=3$. Schöning's algorithm makes use of randomness in the following two parts: (i) choosing initial assignments for local search uniformly at random, and (ii) performing random walks as the local search. Dantsin et al. [5] derandomized it (i) by constructing a set of Hamming balls (so-called covering codes), which efficiently covers the entire search space $\{0,1\}^{n}$, and (ii) by replacing each random walk by backtracking search. Here (i) is "perfectly" derandomized in some sense, however, the derandomization of (ii) loses some efficiency. For 3-SAT, the efficiency in derandomizing part (ii) was gradually improved by a sequence of works $[5,3,21,11]$. Finally, and very recently, Moser and Scheder [13] showed 
a full derandomization of Schöning's algorithm, that is, they proposed a deterministic algorithm for $k$-SAT that runs in time $\widetilde{O}\left((2-2 / k+\epsilon)^{n}\right)$ for any $\epsilon>0$. The running time matches that of Schöning's algorithm, and we now have a deterministic $\widetilde{O}\left(1.3334^{n}\right)$ time algorithm for 3-SAT.

\section{Our Contribution}

We investigate the possibility of derandomizing faster randomized algorithms for 3-SAT. In [8], Hofmeister, Schöning, Schuler and Watanabe improved Schöning's algorithm for the 3-SAT case, that is, they proposed a randomized algorithm for 3-SAT that runs in expected time $\widetilde{O}\left(1.3303^{n}\right)$. Their improvement is based on a sophisticated way of randomly choosing initial assignments rather than just choosing the ones uniformly at random.

In this paper, we present a full derandomization of their algorithm, that immediately implies the following result:

Theorem 1. Problem 3-SAT is deterministically solvable in time $\widetilde{O}\left(1.3303^{n}\right)$.

As long as the authors know, it is the currently fastest deterministic algorithm for 3-SAT. Our result seems to be a necessary step towards a full derandomization of the currently best known randomized algorithm, since it is based on the combination of two algorithms [9] and [7], which are respectively a modification of Hofmeister et al.'s algorithm [8] and an extension of Paturi et al.'s algorithm [15].

To prove the above result, we develop a new way of explicitly constructing covering codes with the properties which corresponds to the distribution used to generate initial assignments in Hofmeister et al.'s algorithm.

More precisely, we respectively denote by SCH and HSSW the randomized algorithms by Schöning [20], and by Hofmeister, Schöning, Schuler, and Watanabe [8]. Algorithm HSSW is obtained by modifying SCH, where one of the main differences between SCH and HSSW is to choose initial assignments for random walks as the local search: HSSW starts the random walk at an assignment chosen randomly from $\left(\{0,1\}^{3} \backslash 0^{3}\right)^{\hat{m}}$ for some $\hat{m} \leq n / 3$, while $\mathrm{SCH}$ starts it at an assignment chosen uniformly from the whole space $\{0,1\}^{n}$.

We derandomized this random selection of initial assignments for HSSW in the similar way to $\mathrm{SCH}$ [5], i.e., by constructing a covering code (i.e., a set of balls that covers the whole search space $\left.\left(\{0,1\}^{3} \backslash 0^{3}\right)^{\hat{m}}\right)$. However, due to the difference of $\left(\{0,1\}^{3} \backslash 0^{3}\right)^{\hat{m}}$ and $\{0,1\}^{n}$, we cannot directly apply a uniform covering code developed in [5]. To efficiently cover the space $\left(\{0,1\}^{3} \backslash 0^{3}\right)^{\hat{m}}$, we introduced a generalized covering code, an $[\ell]$-covering code, which is a sequence of codes $C(0), C(1), \ldots, C(\ell)$ such that (i) $C(i)$ is a set of balls of radius $i$, and (ii) $\bigcup_{i=0}^{\ell} C(i)$ covers $\left(\{0,1\}^{3} \backslash 0^{3}\right)^{\hat{m}}$. We remark that the generalized covering code has non-uniform covering radius while an ordinary covering code has uniform radius.

We first show the existence of small $[\ell]$-covering code $(C(0), C(1), \ldots, C(\ell))$, and then similarly to [5], by using an approximation algorithm for the set cover problem, we show a deterministic construction of an $[\ell]$-covering code $\tilde{C}(0), \tilde{C}(1), \ldots, \tilde{C}(\ell)$ such that $|\tilde{C}(i)| \approx$ $|C(i)|$.

We remark that our technique of constructing certain types of covering codes has a potential application, for example, it can be applied to the further extensions $[2,18]$ of HSSW. 


\section{Preliminaries}

In this section, we briefly review HSSW algorithm for 3-SAT proposed in [8]. In what follows, we focus on 3-CNF formulas. Let $\varphi$ be a 3 -CNF formula over $X=\left\{x_{1}, \ldots, x_{n}\right\}$. We alternatively regard $\varphi$ as the set of clauses of $\varphi$. Thus, the size of $\varphi$, which is the number of clauses of $\varphi$, is denoted by $|\varphi|$. For any sub-formula $\varphi^{\prime} \subset \varphi$ (resp., any clause $C \in \varphi$ ), we denote by $X\left(\varphi^{\prime}\right)$ (resp., $X(C)$ ) the set of variables of $\varphi^{\prime}$ (resp., $C$ ).

A clause set $\varphi^{\prime} \subset \varphi$ is independent if $C \cap C^{\prime}=\emptyset$ for any pair of clauses $C, C^{\prime} \in \varphi^{\prime}$. An independent clause set $\varphi^{\prime}$ is maximal if for any clause $C \in\left(\varphi \backslash \varphi^{\prime}\right)$ there exists a clause $C^{\prime} \in \varphi^{\prime}$ such that $C \cap C^{\prime} \neq \emptyset$. For any partial assignment $t$ to $X(\varphi)$, we denote by $\left.\varphi\right|_{t}$ a sub-formula obtained from $\varphi$ by fixing variables according to $t$. Given a 3-CNF formula $\varphi$, algorithm HSSW starts with arbitrarily finding a maximal independent clause set of $\varphi$.

Fact 1 Let $\varphi$ be a 3-CNF formula. Let $\varphi^{\prime} \subset \varphi$ be a maximal independent clause set of $\varphi$. Then, for any assignment $t$ to $X\left(\varphi^{\prime}\right)$, the formula $\left.\varphi\right|_{t}$ is a 2-CNF formula.

Before describing HSSW, we briefly review SCH algorithm for $k$-SAT proposed in [20]. Algorithm SCH is a randomized algorithm which repeats the following procedure exponentially (in $n$ ) many times: choose a random assignment $t$, and run a random walk starting at $t$ as follows: for a current assignment $t^{\prime}$, if $\varphi$ is satisfied by $t^{\prime}$, then output YES and halt. Otherwise, choose an arbitrary clause $C$ unsatisfied by $t^{\prime}$, and then update $t^{\prime}$ by flipping the assignment of a variable of $C$ chosen uniformly at random. This random walk procedure denoted by $\operatorname{SCH}-\mathrm{RW}(\varphi, t)$ is also exploited in HSSW. The success probability of $\operatorname{SCH}-\operatorname{RW}(\varphi, t)$ for a satisfiable $\varphi$ was analyzed in [20]: Let $\varphi$ be a $3-\mathrm{CNF}$ formula that is satisfiable. Let $t_{0}$ be an arbitrary satisfying assignment of $\varphi$. Then, for any initial assignment $t$ with Hamming distance $d\left(t_{0}, t\right)=r$, we have

$$
\operatorname{Pr}\{\operatorname{SCH}-\mathrm{RW}(\varphi, t)=\mathrm{YES}\} \geq\left(\frac{1}{2}\right)^{r} \cdot \frac{1}{\operatorname{poly}(n)} .
$$

Now, we are ready to present HSSW. Given a 3-CNF formula $\varphi$, HSSW first obtains a maximal independent clause set $\varphi^{\prime} \subset \varphi$. Note here that the formula $\left.\varphi\right|_{t}$ for any assignment to $X\left(\varphi^{\prime}\right)$ is a 2 -CNF, and hence we can check in polynomial time whether $\left.\varphi\right|_{t}$ is satisfiable. From this observation, when $\varphi^{\prime}$ is small, we can significantly improve the whole running time, that is, it only requires $\widetilde{O}\left(7^{\mid \varphi^{\prime}} \mid\right)$ time. On the other hand, when the size of $\varphi^{\prime}$ is large, we repeatedly apply the random walk procedure $\mathrm{SCH}-\mathrm{RW}$. In this case, we can also reduce the running time by smartly choosing initial assignments from satisfiable assignments of $\varphi^{\prime}$ : Recall that SCH uniformly chooses initial assignments from $\{0,1\}^{n}$, which utilizes no information on $\varphi$. Intuitively, HSSW uses initial assignments for SCH-RW that are closer to any satisfiable assignment. In fact we can prove that the larger the size of $\varphi^{\prime}$ is, the higher the probability that the random walk starts at an assignment closer to a satisfying assignment is.

Formally, algorithm HSSW is described in Fig. 1. The algorithm contains 5 parameters $\alpha, c$, and triple $\left(p_{1}, p_{2}, p_{3}\right)$ with $3 p_{1}+3 p_{2}+p_{3}=1$. These parameters are set to minimize the whole expected running time.

Consider algorithm HSSW in Fig. 1 when $\left|\varphi^{\prime}\right|>\alpha n$. Let HSSW-RW $\left(\varphi^{\prime}\right)$ be the procedure that is repeated $c$ times. Then, by using the lower bound (1), and setting parameters 
$\operatorname{HSSW}(\varphi) \quad / / \varphi$ : a 3 -CNF formula over $X$

Obtain a maximal independent clause set $\varphi^{\prime} \subset \varphi$

If $\left|\varphi^{\prime}\right| \leq \alpha n$, then

for each $t \in\{0,1\}^{X\left(\varphi^{\prime}\right)}$ that satisfies $\varphi^{\prime}$

Check the satisfiability of $\left.\varphi\right|_{t} \quad / /\left.\varphi\right|_{t}$ : a 2-CNF formula

If $\left|\varphi^{\prime}\right|>\alpha n$, then

$c$ times do

Run $t=$ init-assign $\left(X, \varphi^{\prime}\right)$

Output NO

$\operatorname{Run} \operatorname{SCH}-\operatorname{RW}(\varphi, t)$

init-assign $\left(X, \varphi^{\prime}\right) \quad / /$ return an assignment $t \in\{0,1\}^{X}$ defined as follows

for each $C \in \varphi^{\prime}$

Assume $C=x_{i} \vee x_{j} \vee x_{k}$

Choose a random assignment $t$ to $x=\left(x_{i}, x_{j}, x_{k}\right)$

following the probability distribution:

$$
\begin{aligned}
& \operatorname{Pr}\{x=(1,0,0)\}=\operatorname{Pr}\{x=(0,1,0)\}=\operatorname{Pr}\{x=(0,0,1)\}=p_{1} \\
& \operatorname{Pr}\{x=(1,1,0)\}=\operatorname{Pr}\{x=(1,0,1)\}=\operatorname{Pr}\{x=(0,1,1)\}=p_{2} \\
& \operatorname{Pr}\{x=(1,1,1)\}=p_{3}
\end{aligned}
$$

for each $x \in X \backslash X\left(\varphi^{\prime}\right)$

Choose a random assignment $t$ to $x \in\{0,1\}$

Fig. 1. Algorithm HSSW

$\left(p_{1}, p_{2}, p_{3}\right)$ suitably (c.f., Lemma 1 below), we have: for any satisfiable 3-CNF formula $\varphi$,

$$
\operatorname{Pr}_{t, \mathrm{SCH}-\mathrm{RW}}\left\{\operatorname{HSSW}-\mathrm{RW}\left(\varphi^{\prime}\right)=\mathrm{YES}\right\} \geq\left(\frac{3}{4}\right)^{n} \cdot\left(\frac{64}{63}\right)^{\left|\varphi^{\prime}\right|} .
$$

The whole expected running time $\widetilde{O}\left(1.3303^{n}\right)$ is obtained by setting $\alpha$ to satisfy the following equation.

$$
\left(\left(\frac{3}{4}\right)^{n} \cdot\left(\frac{64}{63}\right)^{\alpha n}\right)^{-1}=7^{\alpha n}
$$

The values of parameters $\left(p_{1}, p_{2}, p_{3}\right)$ are determined according to the following lemma, which will be used by our derandomization.

Lemma 1 (Hofmeister, Schöning, Schuler, and Watanabe [8]). Let $\varphi$ be a 3-CNF formula that is satisfiable, and let $\varphi^{\prime} \subset \varphi$ be a maximal independent clause set of $\varphi$. Let $t$ be a random (partial) assignment obtained via init-assign $\left(X, \varphi^{\prime}\right)$ and restricted to $X\left(\varphi^{\prime}\right)$. Then, for any (partial) assignment $t_{0} \in\{0,1\}^{X\left(\varphi^{\prime}\right)}$ that satisfies $\varphi^{\prime}$,

$$
\underset{t}{E}\left[\left(\frac{1}{2}\right)^{d\left(t_{0}, t\right)}\right]=\left(\frac{3}{7}\right)^{\left|\varphi^{\prime}\right|} .
$$


There are two types of randomness that are used in HSSW: (1) the random assignment obtained via init-assign, and (2) the random walk of SCH-RW. Fortunately, the latter type of randomness can be (fully) removed by the recent result. (Compare it with the inequality (1).)

Theorem 2 (Moser and Scheder [13]). Let $\varphi$ be a 3-CNF formula that is satisfiable. Let $t_{0}$ be an arbitrary satisfying assignment of $\varphi$. Given an assignment $t$ such that $d\left(t_{0}, t\right)=r$ for a non-negative integer $r$. Then, the satisfying assignment $t_{0}$ can be found deterministically in time $\widetilde{O}\left((2+\epsilon)^{r}\right)$ for any constant $\epsilon>0$.

In the next section, we show that the former type of randomness is also not necessary. It is shown by using covering codes, that is in the similar way to [5]. But, the covering code we make use of is different from ordinary ones. For any positive integer $n$, a code of length $n$ is a subset of $\{0,1\}^{n}$, where each element of a code is called a codeword. A code $C \subset\{0,1\}^{n}$ is called an $r$-covering code if for every $x \in\{0,1\}^{n}$, there exists a codeword $y \in C$ such that $d(x, y) \leq r$. This is the definition of an ordinary covering code. We define a generalization of covering codes in the following way:

Definition 1. Let $\ell$ be a non-negative integer. A sequence $C(0), C(1), \ldots, C(\ell)$ of codes is a $\{0,1, \ldots, \ell\}$-covering code, or simply an $[\ell]$-covering code, if for every $x \in\{0,1\}^{n}$, there exists a codeword $y \in C(r)$ for some $r: 0 \leq r \leq \ell$ such that $d(x, y) \leq r$.

For ordinary covering codes, it is easy to show the existence of a "good" $r$-covering code. Moreover, it is known that we can deterministically construct such an $r$-covering code.

Lemma 2 (Dantsin et al. [5]). Let $d \geq 2$ be a divisor of $n \geq 1$, and let $0<\rho<$ $1 / 2$. Then, there is a polynomial $q_{d}(n)$ such that a covering code of length $n$, radius at most $\rho n$, and size at most $q_{d}(n) 2^{(1-h(\rho)) n}$, can be deterministically constructed in time $q_{d}(n)\left(2^{3 n / d}+2^{(1-h(\rho)) n}\right)$.

\section{A derandomization of HSSW}

In this section, we prove Theorem 1 by derandomizing HSSW. We do that in the similar way to [5]. Let $\varphi$ be a 3-CNF formula, and $\varphi^{\prime}$ be a maximal independent clause set of $\varphi$. Let $\left|\varphi^{\prime}\right|=\hat{m}$, and we suppose $\hat{m}=\Omega(n)$. As is explained in the Introduction, we will use a generalized covering code: an $[\ell]$-covering code. First, we show that there exists an $[\ell]$-covering code for $\left(\{0,1\}^{3} \backslash 0^{3}\right)^{\hat{m}}$ where each of its codes is of small size.

Lemma 3. For $\left(\{0,1\}^{3} \backslash 0^{3}\right)^{\hat{m}}$, there exists an $[\ell]$-covering code $C(0), C(1), \ldots, C(\ell)$, where $\ell$ is the maximum integer such that $(3 / 7)^{\hat{m}}<(1 / 2)^{\ell-2}$, and $|C(i)|=O\left(\hat{m}^{2}(7 / 3)^{\hat{m}} / 2^{i}\right)$.

Proof. We show the existence of such an $[\ell]$-covering code by a probabilistic argument, as is the case of the existence of an ordinary covering code for $\{0,1\}^{n}$. However, the probabilistic construction of an $[\ell]$-covering code is different from the simple one of an ordinary covering code in terms of, (1) non-uniform covering radius, and (2) non-uniform choice of codewords. 
For obtaining the desired covering code, we make use of the probability distribution calculated in [8], that is, the equation (3) of Lemma 1. The probabilistic construction is as follows: Let $\ell$ be the integer defined above. For each $i: 0 \leq i \leq \ell$, let $C(i) \subset$ $\left(\{0,1\}^{3} \backslash 0^{3}\right)^{\hat{m}}$ be a random code obtained by choosing $y \in\left(\{0,1\}^{3} \backslash 0^{3}\right)^{\hat{m}}$ according to the distribution defined by the function init-assign (in Fig. 1), and by repeating it independently $8 \hat{m}^{2}(7 / 3)^{\hat{m}} / 2^{i}$ times.

We will show that $C(0), C(1), \ldots, C(\ell)$ is an $[\ell]$-covering code with high probability. Fix $x \in\left(\{0,1\}^{3} \backslash 0^{3}\right)^{\hat{m}}$ arbitrarily. Note here that $\ell \leq 2 \hat{m}$ and $(1 / 2)^{\ell-1} \leq(3 / 7)^{\hat{m}}$. Then,

$$
\begin{aligned}
& \sum_{i=0}^{3 \hat{m}}(1 / 2)^{i} \operatorname{Pr}_{y}\{d(x, y)=i\} \\
= & \sum_{i=0}^{\ell}(1 / 2)^{i} \operatorname{Pr}_{y}\{d(x, y)=i\}+\sum_{i=\ell+1}^{3 \hat{m}}(1 / 2)^{i} \operatorname{Pr}_{y}\{d(x, y)=i\} \\
\leq & \sum_{i=0}^{\ell}(1 / 2)^{i} \operatorname{Pr}_{y}\{d(x, y)=i\}+(1 / 2)^{\ell} \\
\leq & \sum_{i=0}^{\ell}(1 / 2)^{i} \operatorname{Pr}_{y}\{d(x, y)=i\}+(3 / 7)^{\hat{m}} / 2 .
\end{aligned}
$$

Recall from the equation (3) of Lemma 1 that,

$$
\underset{y}{\mathrm{E}}\left[\left(\frac{1}{2}\right)^{d(x, y)}\right]=\sum_{i=0}^{3 \hat{m}}(1 / 2)^{i} \operatorname{Pr}_{y}\{d(x, y)=i\}=\left(\frac{3}{7}\right)^{\hat{m}} .
$$

From these two, we have

$$
\sum_{i=0}^{\ell}(1 / 2)^{i} \operatorname{Pr}_{y}\{d(x, y)=i\} \geq(3 / 7)^{\hat{m}} / 2 .
$$

From this, we see there exists an $r: 0 \leq r \leq \ell$ such that

$$
\operatorname{Pr}_{y}\{d(x, y)=r\} \geq(3 / 7)^{\hat{m}} 2^{r-1} / \ell .
$$

Note that this value of $r$ depends on $x$. Thus, for each $x \in\left(\{0,1\}^{3} \backslash 0^{3}\right)^{\hat{m}}$, if we define

$$
r_{x} \stackrel{\text { def }}{=} \arg \max _{i: 0 \leq i \leq \ell}\left\{(1 / 2)^{i} \operatorname{Pr}\{d(x, y)=i\}\right\}
$$

we see that $r=r_{x}$ satisfies the above inequality (4) ${ }^{4}$. Let $B(z, i)$ be the set of $w \in\{0,1\}^{3 \hat{m}}$ such that $d(z, w) \leq i$. Then, from the lower bound (4), the probability that $x$ is not covered with any $C(i)$ is

$$
\operatorname{Pr}\left\{x \notin \bigcup_{i=0}^{\ell} \bigcup_{z \in C(i)} B(z, i)\right\} \leq \operatorname{Pr}_{C\left(r_{x}\right)}\left\{x \notin \bigcup_{z \in C\left(r_{x}\right)} B\left(z, r_{x}\right)\right\}
$$

\footnotetext{
${ }^{4}$ This definition of $r_{x}$ is not meaningful if we merely show the existence. However, it is used when we consider a deterministic construction. See the next lemma.
} 


$$
\begin{aligned}
& =\operatorname{Pr}_{C\left(r_{x}\right)}\left\{\forall y \in C\left(r_{x}\right)\left[d(x, y)>r_{x}\right]\right\} \\
& =\left(\operatorname{Pr}_{y}\left\{\left[d(x, y)>r_{x}\right]\right\}\right)^{\left|C\left(r_{x}\right)\right|} \\
& =\left(1-\operatorname{Pr}_{y}\left\{d(x, y) \leq r_{x}\right\}\right)^{\left|C\left(r_{x}\right)\right|} \\
& \leq\left(1-\operatorname{Pr}_{y}\left\{d(x, y)=r_{x}\right\}\right)^{\left|C\left(r_{x}\right)\right|} \\
& \leq\left(1-(3 / 7)^{\hat{m}} 2^{r_{x}-1} / \ell\right)^{\left|C\left(r_{x}\right)\right|} \\
& \leq \exp \left(-(3 / 7)^{\hat{m}} 2^{r_{x}-1}\left|C\left(r_{x}\right)\right| / \ell\right) \\
& \leq \exp (-2 \hat{m})
\end{aligned}
$$

Thus, from the union bound, the probability that some $x \in\left(\{0,1\}^{3} \backslash 0^{3}\right)^{\hat{m}}$ is not covered with any $C(i)$ is at most $7^{\hat{m}} \cdot \exp (-2 \hat{m})=o(1)$. Therefore, there does exist an $[\ell]$-covering code stated in this lemma.

Note that this lemma only shows the existence of such an $[\ell]$-covering code. We need to deterministically construct it. However, we can get around this issue in the same way as [5]: applying the approximation algorithm for the set cover problem. But, since an $[\ell]$-covering code is not of uniform radius, we can not directly apply the approximation algorithm.

Lemma 4. Let $d \geq 2$ be a constant that divides $\hat{m}$, and let $\hat{m}^{\prime}=\hat{m} / d$. Let $\ell^{\prime}$ be the maximum integer such that $(3 / 7)^{\hat{m}^{\prime}}<(1 / 2)^{\ell^{\prime}-2}$, and let $s_{i}^{\prime}=8 \hat{m}^{\prime 2}(7 / 3)^{\hat{m}^{\prime}} / 2^{i}$ for each $i: 0 \leq i \leq \ell^{\prime}$. Let $\ell=\ell^{\prime} d$. Then, there is a polynomial $q_{d}(\hat{m})$ that satisfies the following: an $[\ell]$-covering code $C(0), C(1), \ldots, C(\ell)$ for $\left(\{0,1\}^{3} \backslash 0^{3}\right)^{\hat{m}}$ such that $|C(i)| \leq q_{d}(\hat{m}) \cdot(7 / 3)^{\hat{m}} / 2^{i}$ for $0 \leq i \leq \ell$, can be deterministically constructed in time poly $(\hat{m}) \cdot 7^{3 \hat{m} / d}+q_{d}(\hat{m}) \cdot(7 / 3)^{\hat{m}}$.

Proof. First, we deterministically construct an $\left[\ell^{\prime}\right]$-covering code $D^{\prime}(0), D^{\prime}(1), \ldots, D^{\prime}\left(\ell^{\prime}\right)$ for $\left(\{0,1\}^{3} \backslash 0^{3}\right)^{\hat{m}^{\prime}}$ such that $\left|D^{\prime}(i)\right| \leq \operatorname{poly}\left(\hat{m}^{\prime}\right) \cdot s_{i}^{\prime}$. (Then, we concatenate all of them. See below for details.) Recall the proof of the previous lemma: Let $p_{i}=(1 / 2)^{i} \operatorname{Pr}\{d(x, y)=i\}$ for each $i: 0 \leq i \leq \ell^{\prime}$. For any $x \in\left(\{0,1\}^{3} \backslash 0^{3}\right)^{\hat{m}^{\prime}}$, we have defined $r_{x}=\arg \max \left\{p_{i}\right.$ : $\left.0 \leq i \leq \ell^{\prime}\right\}$, which depends only on $x$. Then, we have concluded that the sequence $C^{\prime}(0), C^{\prime}(1), \ldots, C^{\prime}\left(\ell^{\prime}\right)$ of random codes satisfies the following with high probability: every $x \in\left(\{0,1\}^{3} \backslash 0^{3}\right)^{\hat{m}^{\prime}}$ is covered with the random code $C^{\prime}\left(r_{x}\right)$.

Fix such an $\left[\ell^{\prime}\right]$-covering code $C^{\prime}(0), C^{\prime}(1), \ldots, C^{\prime}\left(\ell^{\prime}\right)$ for $\left(\{0,1\}^{3} \backslash 0^{3}\right)^{\hat{m}^{\prime}}$. Note here that $\left|C^{\prime}(i)\right|=s_{i}^{\prime}$ for $0 \leq i \leq \ell^{\prime}$. For each $i: 0 \leq i \leq \ell^{\prime}$, let

$$
A_{i} \stackrel{\text { def }}{=}\left\{x \in\left(\{0,1\}^{3} \backslash 0^{3}\right)^{\hat{m}^{\prime}}: r_{x}=i\right\}
$$

Note that $\left[A_{0}, A_{1}, \ldots, A_{\ell^{\prime}}\right]$ is a partition of $\left(\{0,1\}^{3} \backslash 0^{3}\right)^{\hat{m}^{\prime}}$. Below, we regard that $C^{\prime}(i)$ dedicates to covering (only) $A_{i}$ (although some codeword of $C^{\prime}(i)$ may cover some elements outside $A_{i}$ ).

The point of the proof is that we apply the approximation algorithm for the set cover problem to $A_{i}$ (not to the whole space $\left(\{0,1\}^{3} \backslash 0^{3}\right)^{\hat{m}^{\prime}}$ ), from which we (deterministically) 
obtain a covering code for $A_{i}$. For this, we obtain all elements of $A_{i}$ and keep them. This is done by calculating the value of $r_{x}$ for each $x \in\left(\{0,1\}^{3} \backslash 0^{3}\right)^{\hat{m}^{\prime}}$, Furthermore, the calculation of $r_{x}$ is done by calculating $p_{j}$ for every $j: 0 \leq j \leq \ell^{\prime}$ : enumerate all $y \in\left(\{0,1\}^{3} \backslash 0^{3}\right)^{\hat{m}^{\prime}}$ such that $d(x, y)=j$, and then calculate the probability that $y$ is generated by the function init-assign. Then, summing up those values of the probability, we can calculate $\operatorname{Pr}\{d(x, y)=j\}$, and hence $p_{j}$. Choosing $j$ as $r_{x}$ such that $p_{j}$ is the maximum of all $j: 0 \leq j \leq \ell^{\prime}$, we can obtain the value of $r_{x}$, and hence $A_{i}$. In total, it takes $\operatorname{poly}(\hat{m}) \cdot 7^{2 \hat{m}^{\prime}}$ time for that procedure.

Now, we apply the approximation algorithm for the set cover problem to each $A_{i}$. As is similar to [5], the approximation algorithm finds a covering code $D^{\prime}(i)$ for $A_{i}$ such that $\left|D^{\prime}(i)\right| \leq q\left(\hat{m}^{\prime}\right) \cdot s_{i}^{\prime}$ in time $q\left(\hat{m}^{\prime}\right) \cdot\left|A_{i}\right|^{3}$ for some polynomial $q\left(\hat{m}^{\prime}\right)$. In total, since $\left|A_{i}\right| \leq 7^{\hat{m}^{\prime}}$, it takes $q\left(\hat{m}^{\prime}\right) \cdot 7^{3 \hat{m}^{\prime}}$ time for that procedure.

So far, we have obtained an $\left[\ell^{\prime}\right]$-covering code $D^{\prime}(0), D^{\prime}(1), \ldots, D^{\prime}\left(\ell^{\prime}\right)$ for $\left(\{0,1\}^{3} \backslash 0^{3}\right)^{\hat{m}^{\prime}}$ such that $\left|D^{\prime}(i)\right| \leq \operatorname{poly}\left(\hat{m}^{\prime}\right) \cdot s_{i}^{\prime}$. For each $0 \leq i \leq \ell=\ell^{\prime} d$, let

$$
C(i) \stackrel{\text { def }}{=}\left\{D^{\prime}\left(i_{1}\right) \times D^{\prime}\left(i_{2}\right) \times \cdots \times D^{\prime}\left(i_{d}\right): i=i_{1}+i_{2}+\cdots+i_{d}, 0 \leq i_{j} \leq \ell^{\prime}\right\} .
$$

It is easy to see that $C(0), C(1), \ldots, C(\ell)$ is an $[\ell]$-covering code for $\left(\{0,1\}^{3} \backslash 0^{3}\right)^{\hat{m}}$. We (naively) estimate the upper bound on $|C(i)|$. Let $i_{1}, i_{2}, \cdots, i_{d}$ be integers such that $i=$ $i_{1}+i_{2}+\cdots+i_{d}$ and $0 \leq i_{j} \leq \ell^{\prime}$. Then,

$$
\begin{aligned}
& \left|D^{\prime}\left(i_{1}\right) \times D^{\prime}\left(i_{2}\right) \times \cdots \times D^{\prime}\left(i_{d}\right)\right| \\
= & \left(\operatorname{poly}\left(\hat{m}^{\prime}\right)\right)^{d} \cdot \frac{8 \hat{m}^{\prime 2}(7 / 3)^{\hat{m}^{\prime}}}{2^{i_{1}}} \cdot \frac{8 \hat{m}^{\prime 2}(7 / 3)^{\hat{m}^{\prime}}}{2^{i_{2}}} \cdots \frac{8 \hat{m}^{\prime 2}(7 / 3)^{\hat{m}^{\prime}}}{2^{i_{d}}} \\
= & \left(\operatorname{poly}\left(\hat{m}^{\prime}\right)\right)^{d} \cdot \frac{\left(8 \hat{m}^{\prime 2}\right)^{d}(7 / 3)^{\hat{m}^{\prime} d}}{2^{i_{1}+\cdots+i_{d}}} \\
= & \left(\operatorname{poly}\left(\hat{m}^{\prime}\right)\right)^{d} \cdot \frac{(7 / 3)^{\hat{m}}}{2^{i}} .
\end{aligned}
$$

Since the number of combinations $i_{1}, \ldots, i_{d}$ such that $i=i_{1}+\cdots+i_{d}$ and $0 \leq i_{j} \leq \ell^{\prime}$ is at most $\left(\ell^{\prime}+1\right)^{d}$, we have

$$
|C(i)| \leq\left(\ell^{\prime}+1\right)^{d} \cdot \operatorname{poly}\left(\hat{m}^{\prime}\right) \cdot \frac{(7 / 3)^{\hat{m}}}{2^{i}} \leq q_{d}(\hat{m}) \cdot \frac{(7 / 3)^{\hat{m}}}{2^{i}}
$$

for some polynomial $q_{d}(\hat{m})$.

Finally, we check the running time needed to construct $C(i)$. It takes $q(\hat{m}) \cdot 7^{3 \hat{m} / d}$ time to construct the $\left[\ell^{\prime}\right]$-covering code $D^{\prime}(0), D^{\prime}(1), \ldots, D^{\prime}\left(\ell^{\prime}\right)$ for $\left(\{0,1\}^{3} \backslash 0^{3}\right)^{\hat{m}^{\prime}}$. Furthermore, it takes $\sum_{i=0}^{\ell}|C(i)|$ to construct the $[\ell]$-covering code $C(0), C(1), \ldots, C(\ell)$ for $\left(\{0,1\}^{3} \backslash 0^{3}\right)^{\hat{m}}$, which is at most $q_{d}(\hat{m}) \cdot(7 / 3)^{\hat{m}}$. Summing up, it takes $q(\hat{m}) \cdot 7^{3 \hat{m} / d}+q_{d}(\hat{m}) \cdot(7 / 3)^{\hat{m}}$ in total.

Recall that $\left|\varphi^{\prime}\right|=\hat{m}=\Omega(n)$. Let $n^{\prime}=n-3 \hat{m}$, which is the number of variables in $\varphi$ not appeared in $\varphi^{\prime}$. For the space $\{0,1\}^{n^{\prime}}$, we use an ordinary covering code, that is guaranteed by Lemma 2 to be deterministically constructed.

Corollary 1. Let $d$ be a sufficiently large positive constant, and let $0<\rho<1 / 2$. Then, there is a polynomial $q_{d}(n)$ that satisfies the following: an $\left\{i+\rho n^{\prime}: 0 \leq i \leq \ell\right\}$-covering code $C\left(0+\rho n^{\prime}\right), C\left(1+\rho n^{\prime}\right), C\left(2+\rho n^{\prime}\right), \ldots, C\left(\ell+\rho n^{\prime}\right)$ for $\left(\{0,1\}^{3} \backslash 0^{3}\right)^{\hat{m}} \times\{0,1\}^{n^{\prime}}$ such that $|C(i)| \leq q_{d}(n)(7 / 3)^{\hat{m}} 2^{(1-h(\rho)) n^{\prime}} / 2^{i}$, can be deterministically constructed in time $q_{d}(n)(7 / 3)^{\hat{m}} 2^{(1-h(\rho)) n^{\prime}}$. 
Proof. It is derived from the previous lemma and Lemma 2. Given an $[\ell]$-covering code $C_{1}(0), C_{1}(1), \ldots, C_{1}(\ell)$ for $\left(\{0,1\}^{3} \backslash 0^{3}\right)^{\hat{m}}$, and a $\rho n^{\prime}$-covering code $C_{2}\left(\rho n^{\prime}\right)$ for $\{0,1\}^{n^{\prime}}$. For each $0 \leq i \leq \ell$, let

$$
C\left(i+\rho n^{\prime}\right) \stackrel{\text { def }}{=} C_{1}(i) \times C_{2}\left(\rho n^{\prime}\right) .
$$

It is easy to see that $C\left(0+\rho n^{\prime}\right), C\left(1+\rho n^{\prime}\right), C\left(2+\rho n^{\prime}\right), \ldots, C\left(\ell+\rho n^{\prime}\right)$ is an $\left\{i+\rho n^{\prime}\right.$ : $0 \leq i \leq \ell\}$-covering code for the space $\left(\{0,1\}^{3} \backslash 0^{3}\right)^{\hat{m}} \times\{0,1\}^{n^{\prime}}$. Furthermore, $\mid C(i+$ $\left.\rho n^{\prime}\right) \mid \leq q_{d}(n)(7 / 3)^{\hat{m}} 2^{(1-h(\rho)) n^{\prime}} / 2^{i}$ for each $i: 0 \leq i \leq \ell$. From the previous lemma, if the constant $d$ is sufficiently large, the running time for (deterministically) constructing $C_{1}(0), C_{1}(1), \ldots, C_{1}(\ell)$ is at most $q_{d}(\hat{m})(7 / 3)^{\hat{m}}$. Similarly, from Lemma 2, the running time for (deterministically) constructing $C_{2}\left(\rho n^{\prime}\right)$ is at most $q_{d}\left(n^{\prime}\right) 2^{(1-h(\rho)) n^{\prime}}$. Thus, the total running time is at most

$$
\begin{aligned}
& q_{d}(\hat{m})(7 / 3)^{\hat{m}}+q_{d}\left(n^{\prime}\right) 2^{(1-h(\rho)) n^{\prime}}+\sum_{i=0}^{\ell} q_{d}(n)(7 / 3)^{\hat{m}} 2^{(1-h(\rho)) n^{\prime}} / 2^{i} \\
\leq & q_{d}(n)(7 / 3)^{\hat{m}} 2^{(1-h(\rho)) n^{\prime}}
\end{aligned}
$$

for some polynomial $q_{d}(n)$.

Now, using this corollary, we show a derandomization of HSSW, and hence we prove Theorem 1. The outline of the deterministic algorithm is almost same as HSSW, which is described in Fig. 1. We show the derandomization for the case of $\left|\varphi^{\prime}\right|>\alpha n$. Given $\varphi^{\prime}$, we deterministically construct an $\left\{i+\rho n^{\prime}: 0 \leq i \leq \ell\right\}$-covering code $C\left(0+\rho n^{\prime}\right), C(1+$ $\left.\rho n^{\prime}\right), C\left(2+\rho n^{\prime}\right), \ldots, C\left(\ell+\rho n^{\prime}\right)$, as is specified in the proofs of Lemma 2, Lemma 4, and Corollary 1. For any $z \in\{0,1\}^{n}$ and non-negative integer $i$, we denote by $B(z, i)$ the set of $w \in\{0,1\}^{n}$ such that $d(z, w) \leq i$. Then, given such an $\left\{i+\rho n^{\prime}: 0 \leq i \leq \ell\right\}$-covering code, we check whether there is a satisfying assignment within $B\left(z, i+\rho n^{\prime}\right)$ for each $0 \leq i \leq \ell$ and each $z \in C\left(i+\rho n^{\prime}\right)$. It is easy to see that this algorithm finds a satisfying assignment of $\varphi$ if and only if $\varphi$ is satisfiable.

We estimate the running time of the algorithm. For any fixed $i$ and $z$, the search of a satisfying assignment within $B\left(z, i+\rho n^{\prime}\right)$ is done in time $(2+\epsilon)^{i+\rho n^{\prime}}$ for any small constant $\epsilon>0$, which is guaranteed by Theorem 2 . Thus, given an $\left\{i+\rho n^{\prime}: 0 \leq i \leq \ell\right\}$-covering code, the running time for this task for all $B\left(z, i+\rho n^{\prime}\right)$ is at most

$$
\begin{aligned}
& q_{d}(n) \cdot \sum_{0 \leq i \leq \ell}\left(\frac{(7 / 3)^{\hat{m}}}{2^{i}} \cdot 2^{(1-h(\rho)) n^{\prime}}\right) \cdot 2^{i+\rho n^{\prime}} \cdot(1+\epsilon)^{n} \\
= & q_{d}(n) \cdot\left(\frac{7}{3}\right)^{\hat{m}} \cdot\left(2^{(1-h(\rho)) n^{\prime}} \cdot 2^{\rho n^{\prime}}\right) \cdot(1+\epsilon)^{n} \\
= & q_{d}(n) \cdot\left(\frac{7}{3}\right)^{\hat{m}} \cdot\left(\frac{4}{3}\right)^{n^{\prime}} \cdot(1+\epsilon)^{n} \\
= & q_{d}(n) \cdot\left(\frac{4}{3}\right)^{n} \cdot\left(\frac{63}{64}\right)^{\hat{m}} \cdot(1+\epsilon)^{n}, \quad\left(\because n^{\prime}=n-3 \hat{m}\right)
\end{aligned}
$$

for some polynomial $q_{d}(n)$. Note from the above corollary that the running time for constructing $\left\{i+\rho n^{\prime}: 0 \leq i \leq \ell\right\}$-covering code is less than the above value. Thus, the total 
running time in case of $\left|\varphi^{\prime}\right|>\alpha n$ is at most $\widetilde{O}\left((4 / 3)^{n}(63 / 64)^{\hat{m}}(1+\epsilon)^{n}\right)$ for any $\epsilon>0$. (Compare this value with the success probability of (2).) On the other hand, it is easy to see that the running time in case of $\left|\varphi^{\prime}\right| \leq \alpha n$ is at most $\widetilde{O}\left(7^{\hat{m}}\right)$. Therefore, by setting $\alpha$ so that $(4 / 3)^{n}(63 / 64)^{\alpha n}(1+\epsilon)^{n}=7^{\alpha n}$ holds (with $\epsilon>0$ arbitrarily small), we obtain the running time $\widetilde{O}\left(1.3303^{n}\right)$.

\section{Conclusion}

We have shown a full derandomization of HSSW, and thereby present a currently fastest deterministic algorithm for 3-SAT. An obvious future work is to obtain a full derandomization of the currently best known randomized algorithm for 3-SAT [7]. To do so, it seems to be required to derandomize Paturi et al.'s algorithm [15] completely. Another possible future work is to extend HSSW algorithm to the $k$-SAT case. It leads to the fastest deterministic algorithms for $k$-SAT, combined with the derandomization techniques of this paper and Moser and Scheder [13].

\section{References}

1. Manindra Agrawal, Neeraj Kayal, Nitin Saxena, "PRIMES is in P," Annals of Mathematics, 160(2):781-793, 2004.

2. S. Baumer and R. Schuler, "Improving a probabilistic 3-SAT algorithm by dynamic search and independent clause pairs," In Selected Revised Papers of the 6th International Conference on Theory and Applications of Satisfiability Testing (SAT), LNCS 2919, pp. 150-161, 2003.

3. T. Brüeggemann and W. Kern, "An improved deterministic local search algorithm for 3-SAT," Theoretical Computer Science, 329(1-3):303-313, 2004.

4. Karthekeyan Chandrasekaran, Navin Goyal, Bernhard Haeupler, "Deterministic Algorithms for the Lovász Local Lemma," In Proc. of SODA 2010, pp. 992-1004, 2010.

5. E. Dantsin, A. Goerdt, E. Hirsch, R. Kannan, J. Kleinberg, C. Papadimitriou, P. Raghavan, and U. Schöning, "A deterministic $(2-2 /(k+1))^{n}$ algorithm for $k$-SAT based on local search," Theoretical Computer Science, 289(1), pp. 69-83, 2002.

6. E. Dantsin, E. A. Hirsch, "Worst-Case Upper Bounds," In Handbook of Satisfiability, Frontiers in Artificial Intelligence and Applications, Vol. 185, IOS Press, pp. 403-424, 2009.

7. Timon Hertli, Robin A. Moser, Dominik Scheder, "Improving PPSZ for 3-SAT using Crtitical Variables," In Proc. of STACS 2011, to appear.

8. T. Hofmeister, U. Schöning, R. Schuler, and O. Watanabe, "A probabilistic 3-SAT algorithm further improved," In Proc. of the 19th Annual Symposium on Theoretical Aspects of Computer Science (STACS), LNCS 2285, pp. 192-202, 2002.

9. K. Iwama, K. Seto, T. Takai and S. Tamaki, "Improved Randomized Algorithms for 3-SAT," In Proc. of ISAAC 2010, Part I, LNCS 6506, pp. 73-84, 2010.

10. K. Iwama and S. Tamaki, "Improved upper bounds for 3-SAT," In Proceedings of the 15th Annual ACM-SIAM Symposium on Discrete Algorithms (SODA), pp. 328-329, 2004.

11. Konstantin Kutzkov, Dominik Scheder, "Using CSP To Improve Deterministic 3-SAT," arXiv:1007.1166v2, 2010.

12. B. Monien and E. Speckenmeyer, "Solving satisfiability in less than $2^{n}$ steps," Discrete Applied Mathematics 10, pp. 287-295, 1985.

13. R. Moser and D. Scheder, "A Full Derandomization of Schöning's $k$-SAT Algorithm," arXiv:1008.4067v1, 2010, to appear in Proc. of STOC 2011.

14. Sanjeev Mahajan, H. Ramesh, "Derandomizing Approximation Algorithms Based on Semidefinite Programming," SIAM J. Comput., 28(5):1641-1663, 1999.

15. R. Paturi, P. Pudlák, M. Saks, and F. Zane, "An Improve Exponential-Time Algorithm for $k$-SAT," In Proceedings of the 39th Annual IEEE Symposium on Foundations of Computer Science (FOCS), pp. 628-637, 1998. (Journal version: J. of the ACM, 52(3), pp. 337-364, 2005.) 
16. R. Paturi, P. Pudlák, and F. Zane, "Satisfiability coding lemma," In Proceedings of the 38th Annual IEEE Symposium on Foundations of Computer Science (FOCS), pp. 566-574, 1997.

17. Omer Reingold, "Undirected connectivity in log-space," J. ACM, 55(4), Article 17, 2008.

18. D. Rolf, "3-SAT $\in \operatorname{RTIME}\left(O\left(1.32793^{n}\right)\right)$," Electronic Colloquium on Computational Complexity, TR03-054, 2003.

19. D. Rolf, "Improved bound for the PPSZ/Schöning-algorithm for 3-SAT," Journal on Satisfiability, Boolean Modeling and Computation, 1:111-122, 2006.

20. U. Schöning, "A probabilistic algorithm for $k$-SAT and constraint satisfaction problems," In Proceedings of the 40th Annual IEEE Symposium on Foundations of Computer Science (FOCS), pp. 410-414, 1999.

21. D. Scheder, "Guided search and a faster deterministic algorithm for 3-SAT," In Proceedings of the 8th Latin American Symposium on Theoretical Informatics (LATIN), LNCS 4957, pp. 60-71, 2008. 